Dialectologia 23 (2019), 85-113.

ISSN: 2013-2247

Received 10 October 2017.

Accepted 22 January 2018.

\title{
EL DEÍSMO EN ORACIONES COPULATIVAS IDENTIFICATIVAS
}

\author{
Edita GUTIÉRREZ RODRÍGUEZ \\ Universidad Complutense de Madrid * \\ editagutierrez@ucm.es
}

Resumen

En este trabajo se ofrece una descripción del deísmo en oraciones copulativas identificativas del español. En primer lugar, se analiza como sujeto de la oración copulativa el infinitivo con la preposición de que aparece tras la cópula. Tras repasar y precisar los contextos en los que se produce deísmo con verbos predicativos, se ofrecen nuevos datos relevantes sobre esta construcción en las oraciones copulativas, así como una clasificación de los sustantivos que permiten la aparición del deísmo. La comparación muestra que los contextos de deísmo en oraciones con verbo predicativo y con verbo copulativo son similares. Por último, se propone que en estas construcciones, igual que en el resto de las oraciones con deísmo, el segmento de no se comporta como una verdadera preposición, sino que se asimila a una conjunción.

\section{Palabras clave}

subordinación, infinitivos con preposición, copulativas identificativas, copulativas inversas, deísmo

\section{CONSTRUCTIONS WITH <DE + INFINITIVE> (DEÍSMO) IN IDENTIFICATIONAL SENTENCES}

\section{Abstract}

In this paper we present a description of the $<d e+$ infinitive clause $>$ non-standard construction (the so-called deismo) in identificational sentences in Spanish. In the first place, the infinitive with de that appears after the copula is analyzed as the subject of the copulative sentence. After the analysis of the contexts in which this construction occurs with predicative verbs, new relevant data in the context of copulative sentences are offered, as well as a classification of the nouns that allow the appearance of deismo in copulative sentences. The comparison between the two groups shows that generalizations with predicative verbs can be extended to the nouns which are the predicate of identificational sentences.

* Departamento de Lengua Española y Teoría de la Literatura, Facultad de Filología. Universidad Complutense de Madrid. Calle del Profesor Aranguren, s/n 28040 Madrid. 
Finally, it is shown that in these constructions, as in predicative contexts, the segment de does not behave like a preposition but as a complementizer.

\section{Palabras clave}

subordination, infinitives with preposition, copulative sentences, identificational sentences, deísmo

\section{Introducción}

Este trabajo se centra en una construcción que no ha sido estudiada hasta el momento: el deísmo en oraciones copulativas. Se llama deísmo la aparición de la preposición de ante la oración subordinada de infinitivo de ciertos verbos (Di Tullio 2011, 2012; Camus 2013; Benito \& Pato 2015):
a. La FIFA lo prohibió de jugar por un año (México) ${ }^{1}$
b. Me da pena de decir esto pero el terrorismo no es el problema de base (España)

El empleo de la preposición de ante el infinitivo es un patrón de subordinación frecuente en español medieval y clásico, época en la que alternaban la presencia y ausencia de la preposición (ejemplos de Di Tullio 2012: 366):
a. Es convinie de yr se
b. Yo había aceptado de me ir con ellos

Aunque finalmente el patrón sin preposición se generalizó en español, quedaron restos de la antigua construcción con de en variedades dialectales, tanto en España como en América. En español actual, la preposición de aparece ante el infinitivo, tanto si este es objeto (1a) como si es sujeto $(1 b)^{2}$ de verbos predicativos de determinadas

\footnotetext{
${ }^{1}$ Todos los ejemplos que se citan proceden del Corpus del Español: Web/Dialects, a menos que se indique lo contrario. Muchos ejemplos proceden de blogs y se han reproducido tal cual aparecen, incluso con errores de ortografía. En ocasiones los ejemplos se han cortado cuando el contexto no era necesario para la comprensión de este.

${ }^{2}$ En ambos casos, el infinitivo ocupa la posición de argumento interno y aparece, por tanto, pospuesto al verbo.
} 
clases semánticas, como han estudiado Di Tullio (2011, 2012); Camus (2013) y Benito \& Pato (2015). El deísmo se da también cuando el infinitivo es el sujeto de oraciones copulativas identificativas, construcción en la cual el deísmo no ha sido analizado hasta el momento:

(3) a. Queda por establecer que su intención fue de confesar un crimen (Panamá)

b. El mundial será una competencia muy fuerte pero mi objetivo es de estar en el podio, comentó Culzón (República Dominicana)

Como en los casos de deísmo con verbos predicativos, en las oraciones copulativas identificativas la preposición de se puede eliminar de los ejemplos de (2) sin que haya una diferencia apreciable de significado, al menos según los hablantes consultados, procedentes de Castilla-La Mancha:

(4) a. Queda por establecer que su intención fue confesar un crimen

b. El mundial será una competencia muy fuerte pero mi objetivo es estar en el podio, comentó Culzón

La preposición de no aparece ante oraciones subordinadas con todos los verbos predicativos, sino solo con ciertas clases semánticas de verbos. El hecho de que la construcción no pertenezca al estándar influye, sin duda, en la variación e inestabilidad de los datos. De la misma manera, la preposición de no aparece en todas las oraciones copulativas identificativas, sino solo en las que contienen ciertos sustantivos en el predicado. Con algunos nombres, el deísmo no es posible o, al menos, no se encuentran ejemplos en los corpus consultados:

(5) a. el destino de viajar por el mundo

b. Su destino es viajar por el mundo

c. *Su destino es de viajar por el mundo 
A pesar de que el sustantivo destino toma un complemento con la preposición de, como se ve en (5a), cuando aparece en oraciones copulativas la única opción posible es que la oración subordinada sustantiva aparezca sin preposición, de manera que (5c) es agramatical. Nuestro objetivo principal es, por tanto, analizar las oraciones copulativas con deísmo y delimitar los contextos en que esta construcción con de puede aparecer.

El trabajo se organiza de la manera siguiente. Tras la introducción y la explicación de la procedencia de los datos en los $\S 1$ y $\S 2$, en el $\S 3$ se muestra que la construcción que estamos estudiando es un tipo de copulativa identificativa inversa, en la que el infinitivo con de es el sujeto de la construcción. En el $\S 4$ repasamos los contextos que tradicionalmente se han estudiado para el deísmo con verbos predicativos y aportamos nuevos datos que matizan y amplían la información sobre la extensión del deísmo. En el $\S 5$ describimos los tipos de nombres que pueden aparecer como predicado en oraciones copulativas con deísmo y comparamos estos con los que aparecen en oraciones con verbos predicativos. Veremos que la distribución en las oraciones copulativas se corresponde aproximadamente con la del deísmo con verbos predicativos. Por último, en el $\S 6$ mostraremos que el segmento de en nuestras construcciones no se comporta como una verdadera preposición, por lo que se podría extender a las oraciones copulativas el análisis como conjunción que ya se ha ofrecido para los casos con verbo predicativo.

\section{Procedencia de los datos}

Los datos de corpus empleados en este trabajo proceden en su mayoría del Corpus del Español: Web/Dialects de Davies y del Corpus del Español del siglo XXI (CORPES) de la Real Academia Española. El corpus de Davies contiene textos de más de dos millones de páginas web. El hecho de que la procedencia de los textos sea internet hace que se puedan encontrar datos de blogs, más cercanos al lenguaje oral y coloquial. Este tipo de corpus es especialmente útil para una primera aproximación a los fenómenos dialectales $y$, de hecho, hemos encontrado en él ejemplos que no aparecían en los corpus de la Real Academia Española, por ejemplo, con un porcentaje más bajo de textos orales. 
Los juicios de la introspección empleados provienen todos de hablantes de Castilla-La Mancha, una de las zonas deístas del sur de España. El objetivo de este trabajo, sin embargo, no es elaborar un estudio cuantitativo ni señalar áreas geográficas con detalle, sino hacer una primera descripción general del fenómeno del deísmo en oraciones copulativas y explicitar los contextos en los que este se produce.

\section{Las oraciones copulativas identificativas}

Entre las oraciones copulativas, se suelen distinguir dos grandes tipos de construcciones (para el español, véanse Navas Ruiz 1977; Gutiérrez Ordóñez 1986; Fernández Leborans 1991-1992); Fernández Leborans 1999; RAE-ASALE 2009: § 37, 38, entre muchos otros; para un estado de la cuestión sobre las oraciones copulativas, Moro 2006): ${ }^{3}$
a. Oraciones copulativas de caracterización o adscriptivas
b. Oraciones copulativas de identificación, identificativas o ecuativas

Las oraciones copulativas de caracterización son aquellas en las que "se expresan características del referente del sujeto" (RAE-ASALE 2009: $\S 37.5 b)$, como en los ejemplos de (6). El atributo de estas oraciones es típicamente un sintagma adjetivo o un sintagma preposicional (6a). También puede ser un sintagma nominal escueto $(6 b, 6 c)$ o un sintagma nominal indefinido $(6 \mathrm{~d}):^{4}$

\footnotetext{
${ }^{3}$ Fernández Leborans (1999: 37.2.1) señala que en la gramática tradicional española no se hacía esta diferencia. En la bibliografía anglosajona, en cambio, se habla de predicational o characterizational sentences y de equative o identificational sentences. En Den Dikken (2006: 1.3) se hace un repaso exhaustivo de las diferentes clasificaciones de las oraciones copulativas.

${ }^{4}$ Existe cierto desacuerdo en torno a si los sintagmas nominales definidos (SD) (con el artículo definido y con un posesivo) pueden ser atributos en copulativas de caracterización. Los SN definidos se sitúan muy arriba en la escala de la referencialidad (Fernández Leborans 1999: 2384) y funcionan con dificultades como atributo de oraciones copulativas. Todo parece indicar, sin embargo, que sí pueden ser atributos caracterizadores. Las oraciones con dos SN definidos tienen dos lecturas, pueden ser predicativas, con el sintagma nominal empleado por sus propiedades intensionales, o bien pueden ser copulativas identificativas, en las que predomina el componente extensional de este sintagma nominal.
} 
(6) a. Mi primo es $\{$ rubio/de Madrid\}

b. Huir de casa no fue decisión suya

c. Ese chico es profesor

d. Su hermana es una psiquiatra estupenda

Las copulativas de caracterización no aceptan la inversión de los dos sintagmas nominales que constituyen la oración copulativa:

(7) a.*Rubio es mi primo

b. *Una psiquiatra estupenda es su hermana

En este tipo de ejemplos, el segundo sintagma nominal solo aparece en posición precopular si está focalizado y tiene prominencia tonal (Fernández Leborans 1999: 2379):

(8) ALCALDE es Juan, no concejal

Las oraciones que vamos a estudiar no son copulativas caracterizadoras sino copulativas de identificación o ecuativas (para el español, véanse Gutiérrez Ordóñez 1986: cap. 3; Fernández Leborans 1991; Fernández Leborans 1999: § 37.3-37.5; RAEASALE 2009: § 37.5j-x; sobre las oraciones copulativas en general, véanse Declerck 1988; Salvi 1991; Moro 1997; Ramos 2002: 14.1.2.2, así como los estados de la cuestión de Den Dikken 2006; Moro 2006 y Heycock 2013). Las copulativas identificativas son oraciones en las que se establece una relación de identidad o identificación entre dos expresiones referenciales, normalmente dos sintagmas nominales definidos:

(9) a. Juan es mi amigo

b. Su vecino es el alcalde

b. Ese es el problema principal

Además de dos sintagmas nominales definidos, estas oraciones también se construyen con un sintagma nominal definido y una oración subordinada sustantiva 
- de infinitivo (10a) o flexionada (10b) - o bien con dos oraciones subordinadas sustantivas (10c):

(10) a. Viajar por el mundo es su deseo

b. Que entrara tanta gente fue el principal problema

c. Querer es poder

En estas oraciones no se ofrece una característica del sujeto sino que se identifican las personas o cosas, o bien se especifica la referencia de un sintagma nominal. Se puede considerar que solo uno de los dos sintagmas nominales es referencial, mientras que el otro posee carácter descriptivo y es una expresión atributiva (Fernández Leborans 1999: 2369). Resulta especialmente complicado diferenciar entre el constituyente del que se predica, o sujeto, y el constituyente que se predica de este, o atributo. Se suele considerar predicado el sintagma nominal que tiene menor capacidad referencial (o bien, mayor contenido predicativo o intensional). Así, los nombres propios, que carecen de contenido intensional, los pronombres personales o los demostrativos están en lo alto de la escala de referencialidad y, por tanto, serán más fácilmente sujeto de predicación de la oración ecuativa en la que se encuentren.

Las oraciones identificativas pueden presentar el llamado orden recto, con el sintagma nominal más referencial ante la cópula y el menos referencial tras la cópula, como en los ejemplos de (10). También presentan el orden inverso, con el sintagma menos referencial ante la cópula, como en (11b):
a. Juan es el alcalde del pueblo (orden recto)
b. El alcalde del pueblo es Juan (orden inverso)

Estas últimas oraciones se denominan copulativas inversas o copulativas especificativas (Fernández Leborans 1999: 2369). Una oración identificativa inversa es una oración copulativa en la que "se revela la identidad del referente específico que conviene a una descripción determinada; el hablante especifica, mediante la expresión 
poscopular, el objeto o entidad al que se extiende la intensión descrita en la expresión precopular" (Fernández Leborans 1999: 37.4):
a. El asesino es Pedro
b. El problema eres tú
c. Su libro favorito es el Quijote
d. Quien llegó tarde fue Luisa

Las oraciones de deísmo que estamos estudiando son un tipo de especificativas, si bien no son las prototípicas:
a. Su deseo era (de) acudir a la cita
b. Su intención fue (de) hacerte daño
c. Su idea fue (de) salir pronto

Las oraciones subordinadas sustantivas, flexionadas o de infinitivo, aparecen tras la cópula en las oraciones especificativas. El carácter argumental y el tipo de denotación de estas oraciones subordinadas parecen entrar en conflicto con la naturaleza predicativa del atributo. Estas oraciones refieren a eventos determinados (Fernández Leborans 1999: 2401; Hernanz 1999: 36.3.2.2) y, en ese sentido, se considera que entran dentro de las categorías referenciales:
a. La idea es ir con ellos
b. Su deseo es acompañarle y enseñarle el lugar
c. La pregunta fue cómo hacerlo

En RAE-ASALE (2009: $\S 37.4 \mathrm{~m}$ ) se considera que las subordinadas sustantivas no ejercen la función de atributo en este tipo de oraciones. ${ }^{5}$ En un ejemplo como EI

\footnotetext{
${ }^{5}$ Señala Fernández Leborans (1999: n. 60) que es especialmente sorprendente el desacuerdo entre los gramáticos sobre la función de la oración sustantiva en las oraciones especificativas. Seco (1972: 119) considera que la oración es el predicado en ejemplos como Mi deseo es que me lo des enseguida. Villalba (2002: 2270) también señala que en oraciones como L'objectiu serà (de) Iluitar 'El objetivo será (de) luchar', la oración de infinitivo es el atributo; en cambio, Alarcos (1994: 327) afirma que la oración que aparece tras la cópula es el sujeto en La verdad es que no me faltaban modelos. Fernández Leborans
} 
problema es que nos faltan medios, a pesar de que la subordinada sustantiva ocupa la posición típica del atributo, se predica de cierto estado de cosas el ser un problema y, por tanto, el atributo es el problema y no Que nos faltan medios:

(15) a. El problema es que nos faltan medios

b. Que nos faltan medios lo es

c. *El problema lo es

La oración subordinada responde a una pregunta con cuál, no con qué, y puede sustituirse por el pronombre masculino ese, no por el neutro eso (RAE-ASALE, 2009: 37.5I):
a. ¿\{Cuál/*qué\} es el problema? Que falta agua
b. El problema es $\{$ ese/*eso $\}$

En resumen, hemos diferenciado en este apartado entre oraciones copulativas de caracterización o adscriptivas y oraciones copulativas de identificación. Hemos analizado los ejemplos que nos ocupan como un tipo de copulativas identificativas, las llamadas copulativas inversas. Finalmente, hemos visto que el sintagma nominal atributo aparece ante la cópula y que la oración subordinada sustantiva realiza la función de sujeto y aparece tras la cópula. El hecho de que estas oraciones refieran a eventos determinados justifica su mayor referencialidad y, por tanto, su análisis como sujeto oracional.

\section{Contextos de aparición del deísmo con verbos predicativos}

En este apartado repasaremos el tipo de predicado con el que el deísmo aparece, para luego comparar, en $\S 5$, con los contextos de deísmo en oraciones copulativas. La descripción del tipo de predicado que puede aparecer con la preposición de coincide

(1999) se inclina asimismo por considerar estas oraciones como sujeto, pues es el elemento más referencial de los dos que intervienen en la relación copulativa. 
básicamente en los distintos autores que han estudiado el deísmo para diferentes áreas geográficas, si bien no todos los grupos de verbos aparecen en todas las áreas y tampoco coinciden en todas las áreas los verbos con los que se produce el fenómeno dentro del mismo grupo semántico.

Los predicados que presentan deísmo se pueden agrupar desde el punto de vista semántico en las siguientes clases (Di Tullio 2011: 180; 2012: 271; Camus 2013: 23-24; ${ }^{7}$ Benito \& Pato 2015: 35): ${ }^{8}$

1. Predicados psicológicos o de afección ${ }^{9}$ que se construyen frecuentemente con un experimentante en dativo y un tema, la oración de infinitivo, que es sujeto posverbal. Entre estos verbos se encuentran apetecer, costar, dar \{pena/lástima/vergüenza/asco/miedo\}, doler, lamentar, pesar o ser una lástima: ${ }^{10}$ ¿Qué fue lo que más te costó de escribir en este género? (Venezuela)

\footnotetext{
${ }^{6}$ Esta autora señala que tanto en español europeo como americano se registran ejemplos de deísmo, en el registro coloquial, con verbos de voluntad e intención (pensar, esperar), de intento (probar), de aceptación (aceptar), de influencia (pedir) y de acaecimiento (ocurrir).

${ }^{7}$ Camus 2013 distingue, para el área de Castilla-La Mancha, las siguientes clases: verbos de afección (pesar), verbos de intención (intentar), verbos de influencia (proponer), algunos verbos de comunicación o aserción (decir) y construcciones de sentido modal con ser (ser necesario).

${ }^{8}$ Benito \& Pato (2015) distinguen: verbos de afección (pesar), construcciones semánticamente impersonales (ser costumbre), verbos de percepción estativos (ver), verbos de manipulación (hacer), verbos de volición e intención (intentar) y verbos modales (poder).

${ }^{9}$ Como señalábamos más arriba, no todos los verbos de cada clase presentan el fenómeno, ni tampoco coinciden los verbos en las mismas áreas geográficas. Así, entre los verbos psicológicos de afección, algunos muy frecuentes como gustar o encantar nunca se construyen con deísmo en Castilla-La Mancha, pero sí en Andalucía.

${ }^{10}$ Estos predicados forman construcciones impersonales o pseudoimpersonales de carácter estativo y cuentan con dos complementos, un dativo experimentante que controla el sujeto del infinitivo y un tema, el infinitivo, que aparece pospuesto al verbo. Algunos también pueden aparecer sin el dativo, con la lectura arbitraria del sujeto del infinitivo. Elvira (2009) explica que las construcciones estativas personales son relativamente recientes y responden a un patrón de retroceso de las construcciones impersonales. En latín, los verbos de experiencia o sentimiento se construían con dativo o acusativo para el experimentante y genitivo el origen o causa del sentimiento. En español medieval, estas construcciones seguían siendo impersonales (Pesol mucho dela su muerte, General Historia, de Elvira 2009: 20) y se generalizó la preposición de para el argumento tema, incluso en los casos en que en latín no se empleaba el genitivo. Con el tiempo, las construcciones dejan de ser impersonales y el argumento tema pasa a ser el sujeto. Esta construcción ya existía en latín tardío y es general en las lenguas romances: fr. Le cinéma me plait, it. Mi piace il cinema, esp. Me gusta el cine).
} 
2. Predicados de voluntad, deseo e intención: se construyen con un infinitivo de complemento directo, cuyo sujeto está ligado por el sujeto del verbo principal. Entre ellos se hallan desear, ${ }^{11}$ esperar, intentar, pensar, procurar o prometer.

(18) Y hasta prometió de comprar me una licuadora para que yo pueda haser jugo en mis horas de ósio... (Argentina)

3. Predicados de influencia y otros predicados que designan actos de habla directivos: se construyen frecuentemente con un infinitivo de complemento directo cuyo sujeto está ligado por el complemento directo del verbo principal. Entre ellos se encuentran decir ('sugerir'), mirar ('intentar'), pedir, permitir, prohibir, proponer o sugerir:

El doctor me prohibió de jugar fútbol pero no le hice caso (Perú)

4. Otras construcciones semánticamente impersonales: se construyen con una oración de infinitivo posverbal que formalmente es el sujeto oracional; el sujeto del infinitivo tiene una interpretación arbitraria (Conviene aprender a cocinar), o bien está ligado por un argumento dativo (Te conviene ir). Algunos de ellos tienen valor modal. Entre los predicados de este grupo se encuentran bastar, convenir, hacer falta, ser \{costumbre/necesario/improbable/fácil\}, tener costumbre, tocar: ${ }^{12}$

(20) La noche anterior, o sea diciembre 7, es costumbre de iluminar con candelas cada parte exterior de las casas (Costa Rica)

Hasta aquí las clases de predicados que, según todos los autores, se construyen con deísmo en todas las áreas del español y en las cuales el deísmo está muy extendido.

\footnotetext{
${ }^{11}$ Solo en la perífrasis estar deseando, que parece parcialmente lexicalizada.

12 Entre estos predicados se encuentran los que en Di Tullio $(2011,2012)$ se denominan verbos de acaecimiento, como ocurrir o pasar. Algunos de estos verbos son los que más habitualmente se construyen con la preposición de.
} 
Vamos a examinar ahora las clases de predicados que se han señalado solo en algunas áreas dialectales.

5. Predicados modales que forman perífrasis: poder, querer, soler. Según Benito y Pato (2015: 38), estos predicados -que no señalan ni Di Tullio $(2011,2012)$ ni Camus (2013)-, se construyen también con deísmo en Extremadura y Andalucía. En nuestra búsqueda en el corpus de Davies, hemos encontrado numerosos ejemplos con los verbos poder y querer en toda América. Con el verbo soler, en cambio, solo hemos encontrado ejemplos en combinación con el verbo ser.

(21) a. Esta dolencia es incapacitante y, si no se trata, puede de originar tofos o alteraciones articulares destructivas (Ecuador)

b. un país que quiere de hacer de sus regiones los pilares y motores del desarrollo (Perú)

c. Es importante tener la teoría a la mano y un libro suele de ser de gran ayuda al momento de hacer esto (México)

En consecuencia, se puede afirmar que en América estos predicados que forman perífrasis modales también se construyen con deísmo; los ejemplos son especialmente frecuentes en México y Centroamérica, y no hemos encontrado ejemplos en el Cono Sur, aunque una investigación más detallada parece necesaria.

6. Predicados de lengua o comunicación: Di Tullio $(2011,2012)$ incluye los verbos de comunicación entre el tipo de verbos que presentan deísmo esporádicamente y señala los siguientes ejemplos: decir, insistir, rogar, solicitar. En cambio, en los datos del COSER de Benito \& Pato (2015), que pertenecen a la Península Ibérica, no hay ejemplos con verbos de comunicación. Estos autores lo atribuyen al tipo de entrevista, que no favorece la aparición de este tipo de verbos, pero también señalan que los ejemplos de Di Tullio podrían estar lexicalizados (decir de venir). En efecto, el verbo decir con la preposición de tiene un significado semejante a proponer y con este sentido no puede prescindir de la preposición ( ${ }^{*}$ Me dijo venir). Este predicado no se incluiría entre los que se construyen con deísmo, pues la preposición parece una preposición regida (cf. Me dijo de su venida).

En nuestra búsqueda en el corpus de Davies hemos encontrado deísmo con algunos de los verbos que señala Di Tullio: 
(22) a. La anfitriona de Aurelia estaba a punto de recibir a unos miembros de su familia y Princesa aceptó de ser también el anfitrión de la chica francesa. (Paraguay)

b. El maletero insistió de llevar mi maleta al cuarto y se tomo 15 minutos en subirla (México)

Sin embargo, los verbos que Di Tullio (2012) señala no parecen ejemplos típicos de verbos de comunicación y algunos como solicitar o rogar encajarían mejor en la clase 3 , de los verbos de influencia. Con otros verbos de comunicación como afirmar, asegurar, gritar, que no se combinan tan fácilmente con infinitivos, se encuentran pocos ejemplos, aunque sí hay alguno:

(23) a. Vas en contra de la seriedad y el debate sano que Elaine dice de tener en su blog (Cuba)

b. El camión fue hallado en el lugar donde Llanca afirmó de haberlo estacionado (Puerto Rico)

En resumen, el deísmo no parece haberse extendido de manera mayoritaria a los verbos de comunicación, ${ }^{13}$ aunque sí se encuentran algunos ejemplos esporádicos.

7. Predicados de percepción: Camus (2013: 25) indica que uno de los contextos en los que más frecuente es el deísmo es el complemento de verbos de percepción y Benito \& Pato (2015: 37) señalan que con los verbos de percepción no agentivos, como ver u oír, se produce deísmo y ofrecen dos ejemplos de Badajoz, España. Di Tullio (2011: 182) señala que este contexto es desconocido en América. ${ }^{14}$ En nuestra búsqueda con los verbos oír y ver hemos encontrado muy pocos ejemplos:

\footnotetext{
${ }^{13}$ Los verbos de comunicación son una de las clases con las que más frecuentemente aparece el dequeísmo, junto con los de creencia. El hecho de que con estas dos clases de verbos no se produzca deísmo sería un argumento para considerar que se trata de fenómenos independientes. Sobre la relación entre deísmo y dequeísmo, véase Di Tullio (2012: 273-275).

${ }^{14}$ En el CORPES se encuentran bastantes ejemplos de deísmo con el verbo ver, tanto en España como en América, en Uruguay: Mañana temprano bajará con ellos y veremos de sacar el coche (España); Un estudio pluviométrico para ver de resolver este tema (Uruguay). Sin embargo, aquí el verbo ver tiene un sentido semejante a 'intentar', es decir, no encajaría entre los verbos de percepción (Di Tullio 2011: 182).
} 
(24)

a. La situación actual se veía de venir (España) $)^{15}$

b. Se los oía de hablar de sus viajes y de los países que habían visto (Cuba)

En nuestra búsqueda en el corpus de Davies hemos encontrado también algún ejemplo con verbos de percepción no estativos, como escuchar:

(25) A Diana Acosta, hoy secretaria de cultura en el Distrito de Barranquilla, le escuché de decir cuando tenía 12 años que sería la periodista que Colombia no había tenido (Colombia)

Sin embargo, con verbos de percepción activos que se construyen frecuentemente con infinitivo como mirar (Le miraba nadar), observar (Te observaba peinarte) o sentir (Le sentía subir por las escaleras) no hemos encontrado ejemplos. En resumen, el deísmo con los verbos de percepción, sean estativos o de significado activo, está poco extendido, con la excepción de ciertas expresiones lexicalizadas.

8. Predicados de pensamiento o juicio: en Di Tullio (2011: 182) se señala que la construcción deísta aparece esporádicamente con verbos de juicio como creer. En nuestra búsqueda hemos encontrado ejemplos con creer, imaginar y con pensar. Con este último verbo la construcción es bastante frecuente y se da tanto en España como en América.

(26) a. Ese Chávez que se cree de venir a mandar a nuestro país nadie le está pidiendo su opinión (Guatemala)

b. Es mejor que sepa que es un cuento que solo usted se lo cree de ser Nóbel de Paz (Nicaragua)

c. Yo pensaba de ponerme un tatuaje de un dragón verde con la lengua afuera echando fuego (España)

${ }^{15}$ Quizá la expresión ver de venir, por ver venir, está parcialmente lexicalizada, al menos en España, donde el verbo ver aparece frecuentemente con deísmo en esta construcción, pero no en otras semejantes como Lo veía de salir. 
9. Predicados causativos: ${ }^{16}$ hacer. Aparte de la expresión hacer de rabiar y hacer de rogar, que están lexicalizadas, hemos encontrado en los corpus otros ejemplos con el verbo hacer:
a. Ya le ha hecho de ponerse a llorar como una magdalena (México)
b. Esa es la filosofía que le ha hecho de llegar a la Liga Endesa desde LEB2 (República Dominicana)
c. No ha triunfado, pero se ha hecho de respetar (España)

En resumen, en este apartado hemos confirmado que las clases de predicados más frecuentes con deísmo son los verbos psicológicos, los de voluntad, los de entendimiento y verbos de distinto tipo semánticamente impersonales. Hemos matizado y ampliado los datos concernientes a los otros tipos de verbos, sobre los que no hay tanto consenso. Hemos visto que el deísmo sí es frecuente con predicados modales como poder y también con el verbo causativo hacer. Por último, aparece esporádicamente con verbos de lengua y comunicación, con los predicados de percepción y con los de pensamiento y juicio.

\section{El deísmo en oraciones copulativas}

En este apartado, vamos a partir de la diferencia entre dos tipos de sustantivos que pueden aparecer en las oraciones copulativas inversas. En primer lugar, las oraciones cuyo predicado es un nombre deverbal y cuyo sujeto es un argumento de este nombre:
a. Su única intención fue de impresionar a sus jefes (México)
b. Su deseo es de ser reconocido por mí (Argentina)

\footnotetext{
${ }^{16}$ La clase que Benito \& Pato (2015: 37) denominan verbos de manipulación, de los que ofrecen dos ejemplos, dejar y hacer. El primero, con el sentido de permitir en los ejemplos que estos autores ofrecen, se podría incluir entre la clase de los predicados de influencia.
} 
En segundo lugar, oraciones copulativas cuyo predicado es un nombre que forma estructuras apositivas y no toma argumentos, como idea, función o planes (Badia 2002: 9.3.2.3; Escandell Vidal 1995: 51; Suñer 1999: 8.4; RAE-ASALE 2009: 12.13o-p, 26.11q).

(29) a. La idea es de acercar mundos (Chile)

b. Aquí la función es de proteger al emprendimiento en sus etapas más tempranas (Venezuela)

A continuación, estudiaremos qué nombres aparecen en el atributo de las oraciones copulativas y si las clases semánticas de sustantivos coinciden con las clases de verbos descritas en el $\S 4$. Como veremos, la relación que se establece entre el primer tipo de nombres y la oración de infinitivo es una relación predicado-argumento, por lo que se espera que las clases coincidan, como realmente ocurre, con ciertas excepciones que también analizaremos. En cambio, la relación semántica entre el segundo tipo de nombre y la oración de infinitivo es más laxa y el deísmo, como veremos, más frecuente con estos sustantivos.

\subsection{El deísmo con sustantivos deverbales}

En ejemplos como los de (28), la oración de infinitivo es un argumento del sustantivo predicado de la oración copulativa, por lo que se puede sostener que hay una relación de predicación entre el sustantivo y la oración de infinitivo, a pesar de que nos encontramos ante oraciones copulativas identificativas. Los sustantivos con los que se produce deísmo coinciden en parte con las clases semánticas de verbos señaladas en el apartado anterior. De los nombres que aparecen a continuación, la mayoría son sustantivos deverbales. Algunos de ellos no lo son, como en (30), pero toman un complemento oracional de infinitivo que desempeña el papel semántico de tema y encajan en las clases semánticas señaladas más arriba:

(30) a. el interés por visitar a sus familiares

b. el miedo a equivocarse 
Dialectologia 23 (2019), 85-113.

ISSN: 2013-2247

1. Predicados psicológicos o de afección: entre los sustantivos relacionados con estos predicados que participan en estructuras deístas se encuentran interés, miedo o preocupación. ${ }^{17}$

(31) a. La verdad que mi principal interés es de contribuir a el fortalecimiento de la cultura ética en el servicio público (El Salvador)

b. Muchas veces el miedo es de quedar atrapado ahí arriba y no poder volver a descender (Paraguay, CORPES) ${ }^{18}$

c. La preocupación es de poder evitar de que afecte a el ducto de gas que va hacia el Brasil y también a las 25 familias de esta comunidad (Bolivia)

2. Predicados de voluntad, deseo e intención: entre los sustantivos que provienen de verbos del tipo señalado, algunos sí se construyen con la preposición de en copulativas identificativas, como deseo, expectativas, intención, promesa, sueño o voluntad. ${ }^{19}$
a. Tengo 4 hijos y tengo 34 años y mi deseo es de adelgazar para sentirme viva otra vez (México)
b. Mi intención era de buscar ese nuevo intento en sus entrañas (Chile, CORPES)
c. Te vas a dar cuenta que por ejemplo, su sueño es de tener una casa grande, o de estar con su familia, viajar (México) ${ }^{20}$

\footnotetext{
${ }^{17}$ Con otros sustantivos, como sorpresa, hemos encontrado solo un ejemplo en los corpus: Mi gran sorpresa fue de descubrir que El parque educativo San José es un zoológico (Colombia). No hemos encontrado ejemplos en los corpus con otros nombres psicológicos como odio, indignación o temor.

${ }^{18}$ El sustantivo miedo toma argumento con la preposición a (el miedo a equivocarse), lo que confirma la idea de que la preposición de que aparece tras la cópula es la típica del deísmo, y no la que acompaña al complemento nominal.

${ }^{19}$ Con otros sustantivos que provienen de verbos de la misma clase semántica, como intento, no hemos encontrado ningún ejemplo en los corpus; de otros sustantivos, solo hemos encontrado un ejemplo, como el nombre esperanza, del que aparece este ejemplo, del español de EE. UU.: Mi esperanza es de compartir con el público general los logros de mi investigación y información asociada. Con el sustantivo promesa solo hemos encontrado un ejemplo: Bien Petras, te agradecemos mucho desde Radio centenario tu participación esta mañana y la promesa es de reencontrarnos el próximo lunes (Colombia).

${ }^{20}$ El sustantivo sueño se acerca aquí al significado del nombre deseo, por eso lo hemos incluido en ese grupo. El verbo soñar se asimila a veces a los sustantivos de percepción o representación, como en Soñé que vivía contigo. Cuando remite a una situación prospectiva, tiene un significado volitivo: Soñé con que iríamos a la playa aquel verano (RAE-ASALE 2009: § 25.40).
} 
d. Parece que la única voluntad que tiene el gobierno es de endosar las causas de la inseguridad a terceros (Venezuela, CORPES)

3. Predicados de influencia: entre los nombres que presentan deísmo relacionados con estos predicados están obligación, orden, propuesta, recomendación. ${ }^{21}$

(33) a. Un medico es un medico, su deber su obligación es de cuidar lo y de atender lo sea cual sea su afiliación política o ideológica (Cuba)

b. Ningún grupo de fuego puede permanecer inactivo esperando ordenes de arriba. Su obligación es de actuar (Honduras)

c. La propuesta es de reconocer los diferentes sistemas de cultivos andinos (Perú)

d. Nuestra recomendación es de hacer lo en la ducha por la mañana antes de la comida (Costa Rica)

4. Nombres derivados de los verbos de significado impersonal. Entre ellos se encuentran acontecimiento, capacidad, costumbre, necesidad, posibilidad o seguridad. ${ }^{22}$ Sin embargo, algunos como acontecimiento (nombre relacionado con los predicados de acaecimiento como ocurrir) no se suelen combinar con oraciones de infinitivo, por lo que es normal que no hayamos encontrado ejemplos de deísmo con él.

(34) a. La costumbre es de comer o tomar sopa de pezcado mientras se toma (Guatemala)

b. Su capacidad es de alterar el espacio / tiempo (México) ${ }^{23}$

c. Buenas tardes. Mi gran necesidad es de tener vivienda propia (México)

d. La única posibilidad es de someterse a la terminación anticipada donde las penas se reducen (Perú)

Hasta aquí podemos confirmar que los sustantivos derivados de verbos se comportan de la misma manera que los verbos con respecto al deísmo. La preposición

\footnotetext{
${ }^{21}$ Con otros sustantivos, como petición, prohibición o sugerencia no hemos encontrado ningún ejemplo en los corpus.

${ }^{22}$ Solo hemos encontrado este ejemplo con el sustantivo seguridad.

${ }^{23}$ Con capacidad solo hemos encontrado este ejemplo.
} 
de aparece en la oración copulativa ante la oración de infinitivo que es sujeto con los sustantivos derivados de los predicados 1-4 del § 4 .

5. Nombres derivados de predicados modales. Los nombres correspondientes a la clase 5 de verbos se encuentran en la anterior clase 4, pues en realidad muchos de los predicados semánticamente impersonales tienen significado modal, y en la clase 5 de los verbos se incluían únicamente aquellos predicados modales que forman perífrasis.

6. Nombres derivados de predicados de lengua o comunicación: no hemos encontrado ejemplos en los corpus de casos de deísmo en oraciones copulativas con sustantivos relacionados con verbos de comunicación, como grito, insistencia, recado, sugerencia o advertencia, ${ }^{24}$ a pesar de que estos sustantivos toman complementos oracionales de infinitivo: su insistencia en ir, su sugerencia de ir, su advertencia de no fumar alli. ${ }^{25}$ Otros sustantivos de esta clase no se suelen construir con infinitivo, como ruego, que lo hace pocas veces (su ruego de volver), o afirmación, que se construye normalmente con oraciones flexionadas (Le sorprendió su afirmación de que la Tierra no se mueve) o con sintagmas nominales. Por otro lado, excepto los casos de decir, que parecen lexicalizados y en los que tiene un significado semejante al del verbo proponer, los ejemplos de deísmo con verbos de lengua o comunicación son escasos, como hemos señalado más arriba. Quizá entra en este grupo un sustantivo como respuesta. ${ }^{26}$

7. Nombres derivados de predicados de percepción: hemos visto arriba que el deísmo con estos verbos es esporádico. Entre los sustantivos que se relacionan con los predicados de percepción, solo sensación parece construirse en oraciones copulativas con de.

\footnotetext{
${ }^{24}$ Algunos de estos sustantivos tienen también un sentido directivo, en el que se acercan a los predicados de influencia.

${ }^{25}$ Hemos encontrado un único ejemplo con el sustantivo advertencia (Se plantearon tres preguntas dirigidas; la advertencia era de centrar se sólo en ellas, evitando las divagaciones) (Chile). El verbo advertir puede ser un verbo de percepción, como en Advirtió que venía 'Vio que venía', pero también un verbo de comunicación, como en Le advirtieron de que no se podía pasar (RAE-ASALE 2009: § 25.3g). El sustantivo advertencia en el ejemplo citado tiene este segundo sentido.

${ }^{26}$ El verbo responder no se construye con oraciones de infinitivo, pero el sustantivo sí (la respuesta de lanzar misiles no gustó). Este infinitivo se corresponde con un gerundio en las estructuras verbales: Respondió lanzando misiles. Tampoco hemos encontrado ejemplos de deísmo en el corpus con este sustantivo.
} 
(35) El conductor se siente compenetrado con el coche casi desde el primer kilómetro y la sensación es de estar manejando un vehículo más grande

Este tipo de ejemplos no parecen del mismo tipo, delante de la preposición se podría incluir un como y la construcción se puede parafrasear como 'la sensación es del estilo de...'.

8. Nombres derivados de predicados de pensamiento y juicio. No hemos encontrado muchos ejemplos. Con el sustantivo creencia (su creencia de ser los mejores) no hay ejemplos en los corpus.

En resumen, igual que ocurre con los verbos, el deísmo se da fundamentalmente con los nombres derivados de predicados psicológicos o de afección, los nombres de voluntad, deseo o intención y los nombres de influencia. Con el resto de los tipos de sustantivos, el deísmo es esporádico.

\subsection{El deísmo con sustantivos de uso apositivo}

También se produce deísmo con ciertos sustantivos abstractos que establecen una relación atributiva con otros sustantivos, tanto en estructuras apositivas con la preposición de (36) como en oraciones copulativas de caracterización e identificación (37):

(36) a. El problema de la droga

'La droga es un problema'

b. El problema de llegar siempre tarde

'Llegar siempre tarde es un problema'

(37) a. El problema es la droga

b. El problema es llegar siempre tarde

Se pueden encontrar en los corpus ejemplos abundantes de deísmo con este tipo de sustantivos: 
(38) a. El objetivo es de instar a los soldados y civiles a que entreguen artículos de contrabando sin ser acosados o penalizados por el gobierno (México)

b. Su problema fue de haber sido una persona infeliz (Chile)

c. La idea es de vender un servicio a un precio fijo (España)

En cambio, con otros sustantivos que forman estructuras apositivas con la preposición de y aceptan oraciones de infinitivo (39), no hemos encontrado ejemplos con deísmo, como aventura, detalle, moda, táctica, tentación ${ }^{27}$ o virtud:

(39) a. la aventura de viajar por el mundo

b. el detalle de conocer a todos por sus nombres

c. la moda de colgar fotos en internet

(40) a. *La aventura es de viajar por el mundo

b. ${ }^{*}$ El detalle es de conocer a todos por sus nombres

d. * La moda es de colgar fotos en internet

En resumen, hemos visto que en las oraciones copulativas identificativas se repite el patrón de aparición de deísmo que hemos analizado con verbos predicativos. Los sustantivos de determinadas clases semánticas, cuando aparecen en el atributo de las oraciones copulativas identificativas permiten la aparición de la preposición de ante la oración de infinitivo que es su sujeto. Además, hemos descrito una clase amplia de sustantivos, que forman relaciones apositivas con sus complementos y que permiten la aparición del deísmo de manera frecuente.

\section{El estatus del segmento de en las construcciones con deísmo}

En diferentes trabajos se ha propuesto que el segmento de no es en estos casos una verdadera preposición, sino una realización del nudo COMP que encabeza las oraciones de infinitivo, es decir, un elemento subordinante (Di Tullio 2011, 2012 o

\footnotetext{
${ }^{27}$ Con el sustantivo táctica hemos encontrado solo un ejemplo, igual que con tentación: su táctica es de fichar a último minuto (Venezuela); La gran tentación es de actuar en forma abusiva (Chile).
} 
Camus 2013 para el deísmo; Villalba 2002 para el catalán; Acquaviva et al. 1991: 1.1-2 y Rizzi 1997 para el italiano). El comportamiento de las estructuras deístas no encaja con el de las oraciones con complemento de régimen cuyo término es una oración de infinitivo.

Diversos contrastes, que repasaremos a continuación, permiten afirmar que en las estructuras deístas con oraciones copulativas que estamos estudiando tampoco hay una verdadera preposición que encabeza un SP cuyo término es una oración de infinitivo ( $\mathrm{Di}$ Tullio 2011, 2012; Camus 2013):

a. Villalba (2002: 2270) argumenta que las oraciones con la preposición de pueden ser sujeto, cosa que no esperamos de un verdadero sintagma preposicional.

b. El complemento encabezado por de se puede pronominalizar por lo cuando es complemento directo, frente a un SP con una preposición regida (acordarse de algo): ${ }^{28}$

(41) a. ¿Mirarás de averiguar esos datos?

b. Sí, lo miraré [Camus 2013: 16]

(42) a. Se acordó de averiguar esos datos

b. *Sí, se lo acordó

En el caso de las oraciones copulativas, el SP se puede sustituir por un pronombre en nominativo sin preposición.
a. Su intención fue de ir
b. Su intención fue esa ${ }^{29}$
c. *Su intención fue de $\{$ esa/eso $\}$

c. La preposición solo aparece con estos verbos cuando el complemento directo es una oración (44a) y (45a), nunca cuando es un sintagma nominal (44b) y (45b), a diferencia de lo que ocurre cuando hay una verdadera preposición (46):

\footnotetext{
${ }^{28}$ De la misma manera, en catalán el infinitivo con de se pronominaliza con el pronombre de acusativo ho y no con el pronombre de genitivo en, que es lo que se esperaría si fuera una verdadera preposición que encabeza un complemento de régimen preposicional (Villalba 2002: 2270).

${ }^{29}$ Agradecemos a nuestros informantes de Castilla-La Mancha todos los juicios de introspección que aparecen en este apartado.
} 
Dialectologia 23 (2019), 85-113.

ISSN: 2013-2247

(44) a. Te dijo de ir

b. *Te dijo de eso

(45) a. El deseo de Juan es de llegar temprano

b. *El deseo de Juan es de su llegada temprana

(46) a. Se acordó de ir

b. Se acordó de eso

d. Cuando un complemento preposicional aparece en una oración hendida, la preposición regida se mantiene (Camus 2013: 20):

(47) a. Me alegro de hacer lo correcto

b. De lo que me alegro es de hacer lo correcto

En los ejemplos con deísmo, en cambio, la preposición no se mantiene:

(48) a. Dije de ir al cine

b. Lo primero que dije es de ir al cine [Camus 2013: 19]

Lo mismo ocurre en las oraciones que estamos estudiando, cuyos juicios de gramaticalidad provienen de hablantes deístas de Castilla-La Mancha:

(49) a. Su deseo es de ser reconocido por mí

b. Lo que es su deseo es de ser reconocido por mí

c. Es de ser reconocido por mí lo que es su deseo

(50) a. Su objetivo es de realizar varios eventos al año

b. Lo que es su objetivo es de realizar varios eventos al año

c. Es de realizar varios eventos al año lo que es su objetivo

e. El infinitivo con la preposición de alterna, en los mismos hablantes, con el infinitivo sin preposición. Esta variación no se da con los verdaderos complementos de régimen preposicional: 
(51) Newton si la acción es empujar, la reacción igual y en sentido contrario será de empujar: el dedo empuja a la piedra y la piedra empuja al dedo. Si la acción es de jalar, entonces la reacción será de jalar: el caballo jala a la piedra y la piedra jala al caballo (Colombia, CORPES)

El infinitivo con la preposición de también alterna con una oración flexionada encabezada por la conjunción que y sin la preposición de:

(52) Su propuesta es que cambie de ciudad o de cambiar su número de teléfono

f. En todos los casos de infinitivo, se puede insertar un artículo delante de la oración de infinitivo con de, lo que prueba que se trata de un contexto nominal -en el que, como es sabido, caben también las oraciones de infinitivo- y no preposicional: ${ }^{30}$
a. Su deseo siempre fue (el) de viajar contigo
b. Su sueño es (el) de tener una casa grande
b. Su intención era (la) de abandonarla

En resumen, en este apartado hemos ofrecido argumentos para mostrar que el segmento de que aparece en las oraciones copulativas identificativas inversas no es una verdadera preposición. Dado que se trata de oraciones de infinitivo que realizan la función de sujeto, se podría aplicar el análisis en el que el segmento de en las

\footnotetext{
${ }^{30}$ Ante las oraciones subordinadas sustantivas de sujeto ( $y$ ante las de $C D$, aunque menos frecuentemente) se puede insertar la forma masculina del artículo determinado (e/), tanto si la subordinada aparece antepuesta como si va pospuesta: El hacer yoga la ayuda a mantenerse en forma; Fue un alivio el llegar a tiempo (Hernanz 1999: 36.3.2.1, Leonetti 1999: 12.1.2.6, RAE-ASALE 2009: XXX). No se deben confundir estos ejemplos con aquellos en que el infinitivo tiene valor nominal: el ir y venir de la gente; cuando el infinitivo es nominal, el artículo es necesario: *ir y venir de la gente. En los ejemplos que estamos estudiando, cuando el de no aparece la presencia del artículo, únicamente masculino, es posible aunque da resultados límites: Su deseo siempre fue el viajar contigo; ?Su sueño es el tener una casa grande; ?Su intención era el abandonarla. En estos casos, el artículo es opcional y, según Leonetti (1999), su presencia depende de la relación semántica que se dé entre la oración de infinitivo y el predicado, de manera que cuanto mayor sea la dependencia semántica del infinitivo respecto del predicado, menor la posibilidad de incluir el artículo. Aun cuando el artículo es opcional, su presencia tiene consecuencias sintácticas, pues el infinitivo aparece inserto en un sintagma nominal. Por ejemplo, impide la extracción del interior de la subordinada, como en las cosas que siento \{haber dicho/*el haber dicho\}.
} 
construcciones deístas (Camus 2013) se corresponde con un complementante reducido (Rizzi 1997), restringido a elementos defectivos como el infinitivo.

\section{Conclusiones y futuras líneas de investigación}

Este trabajo es un primer acercamiento al deísmo en oraciones copulativas inversas, cuyo objetivo fundamental es presentar y organizar nuevos datos de deísmo hasta ahora no estudiados. En primer lugar, hemos repasado las propiedades de las oraciones copulativas de caracterización y de identificación y hemos mostrado que las oraciones que estamos estudiando son copulativas de identificación inversas o especificativas. Este repaso es especialmente relevante porque, al margen del deísmo, las oraciones copulativas de este tipo, en las que el sujeto pospuesto es un argumento del sustantivo que es el predicado de la construcción copulativa han recibido muy poca atención en la bibliografía. En segundo lugar, hemos mostrado que en las oraciones copulativas especificativas se produce deísmo, y que los contextos en los que se produce son semejantes a los de las oraciones predicativas. Los contextos de aparición del deísmo están sujetos a gran variación debido a que se trata de un fenómeno gramatical condenado por la norma y fuera del estándar, lo que dificulta la realización de generalizaciones. A pesar de las dificultades relacionadas con la recogida de datos que no pertenecen al estándar, hemos matizado y ampliado la clasificación de verbos predicativos que presentan deísmo y hemos ofrecido una clasificación detallada de los sustantivos que permiten la aparición de la preposición de ante el infinitivo que es su sujeto. Por último, hemos visto que el comportamiento del segmento de es, en estas oraciones, igual al de las oraciones con verbo predicativo y que se justifica, también en este caso, un análisis formal, que no hemos desarrollado, como conjunción o marca de subordinación ante los infinitivos. Surgen de este trabajo muchas cuestiones que merece la pena seguir investigando, como la relación entre los verbos y las nominalizaciones correspondientes, que dista de ser directa; las diferencias sintácticas entre la lectura apositiva y la argumental de los sustantivos, aquí solo esbozadas; y, por 
último, la relación entre el tipo de nombre y la mayor o menor frecuencia de aparición del deísmo.

\section{Referencias}

AlARCOS LlORACH, E. (1994) Gramática de la lengua española, Madrid: Espasa Calpe.

Acquaviva, P. et al. (1991) "Funzioni delle frasi subordinate", en L. Renzi, G. Salvi \& A. Cardinaletti (eds.), Grande grammatica italiana di consultazione, Bologna: II Mulino, vol. 2, capítulo 13.

BADIA, T. (2002) "Els complements nominals", en J. Solà et alii (dir.), Gramàtica del català contemporani, Barcelona: Empúries, vol. 2, 1591-1640.

Benito, C. de \& E. Pato (2015) "On de + infinitive construction (deísmo) in Spanish", Dialectologia, Special issue, $\mathrm{V}, 29-51$.

<http://www.publicacions.ub.edu/revistes/dialectologiaSP2015/>

Camus, B. (2013) "On deismo. Another Case of Variation in Spanish Complementation", Catalan Journal of Linguistics, 12, 13-39.

DAVIES, M. (2015-2016) Corpus del Español: Web/Dialects, <http://www.corpusdelespanol.org/>

DeCLERK, R. (1988) Studies on Copular Sentences. Clefts and Pseudo-Clefts, Lovaina: Univ. Press, Dordrecht, Foris.

DEN DIKKEN, M. (2006) "Specificational Copular Sentences and Pseudoclefts", en M. Everaert \& H. Van Riemsdijk (eds.), The Blackwell Companion to Syntax, London: Blackwell, vol. IV, 292409.

Di Tuluıo, A. (2011) “Infinitivos introducidos por de”, Cuadernos del ALFAL, 3, 176-187.

Dı TULıIO, A. (2012) "Oraciones completivas de infinitivo introducidas por de en el español moderno", en T. E. Jiménez Juliá et alii (coord.), Cum corde et in nova grammatica: estudios ofrecidos a Guillermo Rojo, Santiago de Compostela: Universidad de Santiago de Compostela, 265-276.

ELVIRA, J. (2009) “Construcciones y significado: aspectos diacrónicos de la transitividad en español", en Seminario internacional. Corrientes de estudio en semántica y pragmática históricas, 23-24 de noviembre de 2009, Madrid: Instituto Menéndez Pidal.

ESCANDELL VIDAL, V. (1995) Los complementos del nombre, Madrid: Arco/Libros.

FERNÁNDEZ LebORANS, M. J. (1991-1992) “Aspectos semánticos y sintácticos de las oraciones identificativas inversas", Dicenda, 10, 73-110. 
FernándeZ Leborans, M. J. (1999) "La predicación: las oraciones copulativas", en I. Bosque \& V. Demonte (dirs.), Gramática descriptiva de la lengua española, Madrid: Espasa, vol. 2, 2357-2460.

GómEZ TORREGO (1999) “La variación en las subordinadas sustantivas: dequeísmo y queísmo”, en I. Bosque \& V. Demonte (dirs.), Gramática descriptiva de la lengua española, Madrid: Espasa, vol. 2, 2105-2148.

GUTIÉRREZ ORDÓÑEZ, S. (1986) Variaciones sobre la atribución, León: Universidad de León.

Hernanz, M. L. (1999) “El infinitivo”, en I. Bosque \& V. Demonte (dirs.), Gramática descriptiva de la lengua española, Madrid: Espasa, vol. 2, 2197-2356.

HeyCOCK, C. (2013) "The Syntax of Predication", en M. den Dikken (ed.), The Cambridge Handbook of Generative Syntax, Cambridge: Cambridge University Press.

LEONETTI, M. (1999), "El artículo", en I. Bosque y V. Demonte (dirs.), Gramática descriptiva de la lengua española, Madrid: Espasa, 787-890.

LeVIN, B. (1993) English Verb Classes and Alternations, Chicago: The University of Chicago Press.

Moro, A. (1997) The Raising of Predicates: Predicative Noun Phrases and the Theory of Phrase Structure, Cambridge: Cambridge University Press.

Moro, A. (2006) "Copular Sentences", en M. Everaert \& H. Van Riemsdijk (eds.) The Blackwell Companion to Syntax, Oxford: Blackwell, vol. 2, 1-25.

NAVAS RUIZ, R. (1977) 'Ser'y 'estar'. El sistema atributivo del español, Salamanca, Almar.

RAE-ASALE (2009) Nueva gramática de la lengua española, Madrid: Espasa.

RAMOS, J. R. (2002) "El SV, II: La predicació no verbal obligatoria", en J. Solà et alii (dirs.), Gramàtica del català contemporani, 2, Barcelona: Empúries, 1951-2044.

REAL ACADEMIA ESPAÑOLA: Banco de datos (CORPES XXI) [en línea]. Corpus del Español del Siglo XXI (CORPES). <http://www.rae.es> [diciembre 2016]

RIzzI, L. (1997) "The Fine Structure of the Left Periphery", en L. L. Haegeman, Elements of Grammar, Kluwer International Handbooks of Linguistics, Dordrecht: Springer, 281-337.

SALVI, G. (1991) "Le frasi copulative”, en L. Renzi, G. Salvi \& A. Cardinaletti (eds.), Grande Grammatica Italiana di Consultazione, Bologna: II Mulino, vol. 2, 163-189.

SUÑER, M. (1999) "La subordinacion sustantiva: La interrogacion indirecta", en I. Bosque \& V. Demonte (dirs.), Gramática descriptiva de la lengua española, Madrid, Espasa, 2149-2196.

VILLALBA, X. (2002) "La subordinació”, en J. Solà et alii (dirs.), Gramàtica del català contemporani, Barcelona: Empúries, vol. 3, 2247-2319. 